\title{
The Direct Economic Burden of Socioeconomic Health Inequalities in Canada: An Analysis of Health Care Costs by Income Level
}

\author{
Social Determinants and Science Integration Directorate, Public Health Agency of Canada
}

Tweet this article

Canadian research indicates that individuals with lower incomes, less education or lower occupational skill levels tend to be less healthy than those who enjoy greater advantages in these areas. ${ }^{1-3}$ This uneven distribution of health across different socioeconomic status (SES) groups is referred to as "socioeconomic inequality in health."

Evidence of the economic cost of health inequalities helps us understand the benefits of reducing these inequalities. However, the data needed to generate such evidence is difficult to obtain. A lack of Canadian data linking health costs and socioeconomic characteristics means that assessment of the degree to which health costs are associated with socioeconomic inequalities at the national level is limited.

In order to build evidence on the cost of socioeconomic health inequalities, the Public Health Agency of Canada worked with Statistics Canada to test the feasibility of a "bottom-up" approach to compiling national health cost data. A bottom-up approach relies on individual-level data, which allows costs to be calculated by individual-level characteristics not always found in other data sources. This includes indicators of SES such as level of education or income. In this study, the population was divided into quintiles based on income, and the health care costs incurred by these five income groups were examined for a single year (2007-2008).
Estimates of health care costs by income level make it possible to assess one dimension of economic impact: the direct economic burden of socioeconomic equalities in health in Canada. The direct economic burden measures the influence of socioeconomic health inequalities on expenditures within the health care system. It represents the estimated reduction in health care costs that could result if all Canadians had the same health care utilization and cost patterns as those in the highest income quintile. ${ }^{\dagger}$

In The Direct Economic Burden of Socioeconomic Health Inequalities in Canada: An Analysis of Health Care Costs by Income Level, ${ }^{4}$ income was used as the proxy measure for SES because data linking health costs to income are more broadly available in Canada than data for other dimensions of SES. However, this approach does not imply that the presence of health care cost differences between income groups is solely due to level of income, or that income (re)distribution is the primary policy lever for reducing health inequalities.

The health care services included in this report were limited to those for which individual-level data were available at the national level, namely acute care inpatient hospitalizations, prescription medications and physician consultations (general practitioner and specialist). Together, these three services represented about onequarter of all health care expenditures in Canada in 2007 to 2008. Expanding available individual-level data would improve the calculation of the direct economic burden of socioeconomic health inequalities.

\section{Highlights}

- Magnitude of the direct economic burden

Socioeconomic health inequalities impose a direct economic burden of at least $\$ 6.2$ billion annually, or over $14 \%$ of total expenditures on acute care inpatient hospitalizations, prescription medication and physician consultations.

- Distribution of the direct economic burden

Canadians in the lowest income group account for $60 \%$ ( $\$ 3.7$ billion) of the total direct economic burden. Improving the health of the lowest SES group could have a significant impact on the costs of socioeconomic health inequalities in Canada.

- Total costs by health care component Total age-standardized costs for the three health care services in this report are $\$ 43.8$ billion. Acute care inpatient hospitalizations make up $50 \%$ of this amount, prescription medications $40 \%$ and physician consultations $10 \% .^{\ddagger}$

\footnotetext{
* There are some exceptions to the use of 2007-2008 as the reference year for the report. For more information, see Section 3 of the full report.

See Figure 4 on page 21 of the full report for an illustration of the way the economic burden of socioeconomic inequalities in health in Canada was calculated.


information, see Section 4 of the full report.

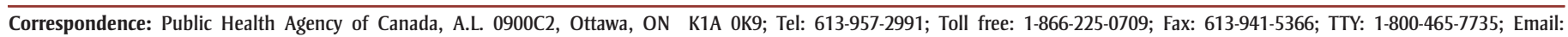
publications@hc-sc.gc.ca 
- Socioeconomic gradient in health care costs

The costs of acute care inpatient hospitalizations and physician consultations generally follow a gradient, meaning that health care costs decline as income rises. Canadians in the lowest income quintile have the highest age-standardized average health care costs.

- Comparing health care cost gradients The difference in health care costs between SES groups is more pronounced between low- and middle- income Canadians than between middle- and highincome Canadians.

- Health care cost patterns by sex Health care costs are generally highest in the lowest income quintile for both women and men.

- Health-adjusted life expectancy by income level

According to the World Health Organization, health-adjusted life expectancy (HALE) is defined as the "average number of years that a person can expect to live in 'full health' by taking into account years lived in less than full health due to disease and/or injury." 5 The more comprehensive HALE data included in this report revealed a socioeconomic gradient: HALE generally declines as income decreases.

\section{Conclusion}

The Direct Economic Burden of Socioeconomic Health Inequalities in Canada: An Analysis of Health Care Costs by Income Level $^{4}$ provides the first national-level estimate of the direct economic burden of socioeconomic inequalities in health in Canada. The burden is an indication of the magnitude of the costs associated with health inequalities-which in turn speaks to the significance of these inequalities for policy development. A better understanding of the direct economic burden can be helpful in considering the balance of health expenditures between prevention and treatment, as well as investments in other important social supports that facilitate healthy lifestyle choices.

\section{References}

1. Canadian Population Health Initiative. Reducing gaps in health: a focus on socioeconomic status in urban Canada. Ottawa (ON): CIHI; 2008.

2. Chief Public Health Officer of Canada. The Chief Public Health Officer's report on the state of public health in Canada: addressing health inequalities. Ottawa $(\mathrm{ON})$ : Public Health Agency of Canada; 2008.

3. McIntosh CN, Finès P, Wilkins R, Wolfson $\mathrm{M}$. Income disparities in health adjusted life expectancy for Canadian adults, 1991-2001. Health Rep. 2009;20(4):55-64.

4. Public Health Agency of Canada. The direct economic burden of socioeconomic health inequalities in Canada: an analysis of health care costs by income level. Ottawa $(\mathrm{ON})$ : Public Health Agency of Canada; 2016.

5. World Health Organization. Health status statistics: mortality [Internet]. Geneva $(\mathrm{CH})$ : World Health Organization; [cited 2016 Apr 11]. Available from: http://www. who.int/healthinfo/statistics/indhale/en/ 